\title{
The grand challenges of the Gates Foundation: what impact on global child health?
}

\author{
Jennifer M Litzow Howard Bauchner
}

J R Soc Med 2006;99:171-174

\section{INTRODUCTION}

Millions of children in poor countries die each year from preventable diseases. ${ }^{1}$ This is not news. Since their inception, international organizations including the World Health Organization (WHO) and the United Nations (UN), have dedicated countless hours and millions of dollars to alleviating this problem. More recently, a number of private foundations have become active in the work of childhood survival. The Bill and Melinda Gates Foundation, one of the leading financial supporters of global health, recently announced over US\$ 450 million to be distributed in grants to 43 projects that address the Foundation's 14 Grand Challenges (Box 1). ${ }^{2}$ The programme aims to stimulate the creation of novel ways to prevent, treat and cure the diseases that kill millions of people each year in developing countries.

In this paper we will explore the potential impact of some of goals of the Grand Challenges for global child health within the context of current international health initiatives including the Integrated Management of Child Diseases created by the WHO and UNICEF, the UN's Millennium Development Goals, and the Bellagio Study Group's assessment of the current state of global child survival in the developing world.

\section{THE CURRENT CONTEXT OF GLOBAL CHILD HEALTH INITIATIVES}

In order to assess the potential impact of the Gates Foundation's Grand Challenges on global child survival, the programme must be considered within the larger context of recent international efforts to improve child health. In the mid-1990s a collaborative effort between the WHO and UNICEF created the Integrated Management of Childhood Illness (IMCI), a strategy which promotes a comprehensive approach to healthcare in developing countries through three components:

1 improving healthcare systems for better delivery of care

2 training of healthcare workers in disease assessment and treatment

Department of Pediatrics, Division of General Pediatrics, Boston University School of Medicine/Boston Medical Center, Boston, MA 02118, USA

Correspondence to: Jennifer M Litzow MD

E-mail: jennifer.litzow@bmc.org

\section{Box 1 Grand Challenges of the Gates Foundation}

- Goal: Improve childhood vaccine

1. Create effective single-dose vaccines that can be used soon after birth

2. Prepare vaccines that do not require refrigeration

3. Develop needle-free delivery systems for vaccines

- Goal: Create new vaccines

4. Devise reliable tests in model systems to evaluate liveattenuated vaccines

5. Solve how to design antigens for effective, protective immunity

6. Learn which immunological responses provide protective immunity

- Goal: Control insects that transmit agents of disease 7. Develop a genetic strategy to deplete or incapacitate a diseasetransmitting insect population

8. Develop a chemical strategy to deplete or incapacitate a disease-transmitting insect population

- Goal: Improve nutrition to promote health

9. Create a full range of optimal, bioavailable nutrients in a single staple plant species

- Goal: Improve drug treatment of infectious diseases

10. Discover drugs and delivery systems that minimize the likelihood of drug resistant microorganisms

- Goal: Cure latent and chronic infection

11. Create therapies that can cure latent infections

12. Create immunological methods that can cure latent infection

- Goal: Measure health status accurately and economically in developing countries

13. Develop technologies that permit quantitative assessment of population health

14. Develop technologies that allow assessment of individuals for multiple conditions or pathogens at point of care

3 improving family and community healthcare practices. ${ }^{3}$

Now introduced in more than 100 countries, IMCI strives to integrate prevention at the household and community levels with the medical treatment of disease. This approach to illness recognizes that multiple factors affect the health of children including low education levels, poor hygiene, inaccessible resources, inadequately trained providers and poorly supplied medical infrastructures. ${ }^{3}$ IMCI has been shown to be somewhat effective in improving child health in countries such as Tanzania and Bangladesh. However, consistent provision of high-quality care and wide spread implementation of IMCI has been difficult to achieve in these and other developing countries, 


\section{Box 2 Millennium development goals}

1. Eradicate extreme poverty and hunger

2. Achieve universal primary education

3. Promote gender equality and empower women

4. Reduce child mortality

5. Improve maternal health

6. Combat HIV/AIDS, malaria and other diseases

7. Ensure environmental sustainability

8. Develop a global partnership for development

primarily due to inadequate financial investments in all three components of the programme. ${ }^{4-6}$

In 2000, the UN announced the Millennium Development Goals (MDGs), a directive to improve the health and wellbeing of children and adults living in the world's poorest countries (Box 2). ${ }^{7}$ All of the MDGs will have a beneficial impact on child health, but one objective is specific to child survival - the reduction of global child mortality by a stunning two-thirds among children under 5 . Unfortunately, without a significant increase in health expenditure in developing countries, this ambitious goal is unlikely to be met. ${ }^{8,9}$ The MDGs created a measurable agenda by which individual countries and organizations may guide efforts to improve child survival; however, the MDGs still exist largely as a symbolic centrepiece of current global child health initiatives. ${ }^{10}$

The effectiveness of these initiatives and the state of child health in the developing world were assessed in 2003 in a series of seminal articles on child survival by the Bellagio Study group. The first article in the series was a sobering reminder that more than 10 million children continue to die in the developing world each year, most commonly from diarrhoea, pneumonia, and malaria. ${ }^{1}$ More than $40 \%$ of the deaths of children under 5 occur during the neonatal period. The authors of this Lancet series argued that these deaths are caused not only by medically preventable diseases but can also be attributed to: the inadequacy of healthcare systems; incomplete epidemiologic knowledge base that hinders our understanding of local health indicators; and the unequal distribution of existing resources. ${ }^{11}$ Therefore, reduction in child mortality relies not just on disease-specific interventions but also on improvements in epidemiology, delivery strategies for child survival and maternal health interventions, programme equity and improvements in programme monitoring. ${ }^{12}$

\section{THE GRAND CHALLENGES}

It is within this context of IMCI, the UN's MDGs and the child survival series that the Bill and Melinda Gates Foundation, in partnership with the National Institutes of Health, announced in June 2005 the recipient grants of the approach to improving global health. In contrast to improving delivery systems of already existing and effective interventions, the Grand Challenges combines financial support from The Gates Foundation (US\$ 450 million), the Wellcome Trust (US\$ 27.1 million), and the Canadian Institutes of Health (US\$ 4.5million) in support of 43 individual projects that each aim to innovatively solve one of the 14 challenges as described by the Gates Foundation (Box 1). ${ }^{13}$ The purpose of the Grand Challenges is to advance science and provide new technologies that will make significant contributions to improving the health of people in developing countries. ${ }^{14}$ Previous to this financial support, only approximately $10 \%$ of the world's medical research was spent on health problems of the developing world. ${ }^{15}$ The Gates Foundation's initiative will champion scientific creativity and support novel ways to curb the diseases of the world's poor. The grants support the creation of new vaccines and the improvement of vaccine delivery capabilities, the improved control of diseasespreading insects, the development of more nutritional food supplies, the development of drugs that are less likely to succumb to microbial resistance, and new diagnostic capabilities for resource-poor settings.

One Grand Challenge is to improve childhood vaccines by the creation of single dose vaccines which are safe for neonates, vaccines that do not require refrigeration, and needle-free vaccine delivery systems. Current vaccine schedules are often difficult and complicated. Prevention of vaccine-preventable diseases remains a global imperative. In 2002, an estimated 1.4 million deaths of children under 5 were from vaccine-preventable diseases. ${ }^{16}$ Immunization coverage of children is often limited by difficulty in accessing multiple doses of vaccines required for full immunity or a breakdown in the cold chain, ruining vaccines that require refrigeration. Most immunizations require needles for administration, exposing children to the risk of transmissible disease through injection. Improved childhood vaccines that allow for broader, safer coverage will directly benefit child health.

Another goal of the Grand Challenges is to create new vaccines against HIV, tuberculosis and malaria. Malaria claims over one million lives every year, most of them children, with substantial additional morbidities. ${ }^{17}$ Mosquito control programmes including insecticide treated bed nets (ITNs) and indoor residual spraying have been a major theme of The Roll-Back Malaria Campaign sponsored by the WHO and other malaria-prevention campaigns. ${ }^{18}$ Cheap and effective medical treatment regimens have also been a focus of these programmes. Despite these interventions, the toll of malaria on child health remains high and available interventions have met with limited success. Nets require re-treatment to remain effectivetoxic insecticides pose a threat to humans - and the malaria 
parasite has been adept at developing resistance to medical treatments. The Grand Challenges address these issues by supporting various grants whose focus is to create a vaccine against malaria, find new genetic and chemical strategies to reduce the ability of the mosquito to transmit disease and develop new medical treatments which withstand the development of microbial resistance. Around the world, most parasites are resistant to chloroquine and sulfadoxinepyrimethamine. Combination therapy is required for adequate treatment of drug-resistant malaria, yet this is extremely costly compared to monotherapy. ${ }^{17}$ Given the toll of malaria on child health, the development of these drugs would have a beneficial impact on child health.

In addition to vaccine-preventable diseases and malaria, malnutrition is recognized as a significant co-morbidity in child health. UNICEF estimates that approximately 150 million children in developing countries are malnourished. Malnutrition is associated with almost one-half of all child deaths worldwide. ${ }^{19}$ Many children suffer illness exacerbated by micronutrient deficiencies. To address this crippling problem, the Grand Challenges will support four separate projects working to create highly nutritious crops including bananas, cassavas, rice and sorghum. Ideally, these genetically engineered crops will not only provide nutritional staples to populations, but will be hearty crops that can flourish in harsh climates, providing relief to areas suffering from hunger caused by inadequate crop plants and environmental factors. Unfortunately, genetically engineered food has not been well received in most countries around the world. We anticipate a long political battle will follow the development of these genetically altered foods.

The ability to determine the exact toll of co-morbidities like malnutrition depends on public health infrastructure and the collection of data at the community level. Too often, public health decisions are based on data that are inaccurate or incomplete for the population at hand. ${ }^{20}$ Towards this end, the Grand Challenges will support one project to create new epidemiologic and health assessment measurement tools which will allow for more precise understanding of health trends. Another grant will support the development of inexpensive and portable diagnostic devices. The extension of diagnostic tools to rural communities will allow healthcare workers to provide medical interventions based on definitive diagnoses.

\section{CHALLENGES OF IMPLEMENTATION}

New and improved vaccines, altered mosquitoes and engineered crops, improved treatment regimens - these are just some of the goals of the Grand Challenges which, if fulfilled, will make significant contributions to child survival. Yet while the projects are innovative and have the potential to save many lives, an entire dimension of global child health is not addressed by the Grand Challenges. Although the ultimate purpose of the project is to create more 'deliverable technologies' ${ }^{21}$ the issues of poor infrastructure, insufficient healthcare delivery systems, and political instability remain significant causes of child mortality and are not addressed nor supported by the Grand Challenges. While science is necessary to advance our understanding of disease and provide new opportunity for treatment and prevention, we cannot permit the intoxicating promise of technology and scientific solution to seduce us from the importance of building social and political systems that work. There is no doubt that with the fulfilment of the Grand Challenges global child health could be improved, but only if the new solutions are distributed and their use effectively implemented. We recognize that indeed the intent of many of the Grand Challenges is to make useful interventions easier to deliver, and we suspect that the Gates Foundation recognizes that widespread, effective implementation is critical to the ultimate success of the new scientific discoveries.

We wait to reap the benefits of future technologies. In order to be truly useful, scientific advancements must be coupled with delivery systems that work. They must be integrated with improved management capabilities, increased infrastructure, and better trained personnel. The Grand Challenges are another step in the process. Improving child survival is a balance between creating new and necessary technology while simultaneously ensuring access and equitable distribution patterns of resources. This may be the grandest challenge of all.

Competing interests Jennifer M Litzow is supported in part by a T32HP-10014 grant. Howard Bauchner is supported in part by an NIH mentoring award K24/ HD042489.

\section{REFERENCES}

1 Black RE, Morris SS, Bryce J. Where and why are 10 million children dying every year? Lancet 2003; 361:2226-34

2 [www.gatesfoundation.org] accessed 29 June 2005

3 [www.who.int/child-adolescent-health/integr.htm] accessed 1 September 2005

4 Bryce J, Victora CG, Habicht J-P, Vaughan P, Black RE. The multicountry evaluation of the integrated management of childhood illness: lessons for the evaluation of public health interventions. Am $J$ Public Health 2004;94:406-15

5 Chellenberg JRA, Adam T, Mshinda H, et al. Effectiveness and cost of facility-based Integrated Management of Childhood Illness (TMCI) in Tanzania. Lancet 2004;364:1583-94

6 Arifeen SE, Blum LS, Hoque DME, et al. Integrated management of childhood illnesses (IMCI) in Bangladesh: early findings from a clusterrandomised study. Lancet 2004;364:1595-1602

7 [www.un.org/millenniumgoals.] Accessed 15 October 2005 
J OUR N A L O F T H E R OYA L S O CIETY OF MEDICINE Volume 99 April 2006

8 Dyer Owen. Some countries will not achieve millennium development goals for health by 2015, WHO says. BMJ 2005;330:863

9 Sachs JD, McArthur JW. The Millennium Project: a plan for meeting the Millennium Development Goals. Lancet 2005;365:347-53

10 Khamsi Roxanne. Can we score the millennium goals? Nature 2005; 437:304

11 Bryce J, el Arifeen S, Pariyo G, Lanata CF, Gwatkin D, Habicht J-P and the Multi-Country Evaluation of IMCI Study Group. Reducing child mortality: can public health deliver? Lancet 2003;362:159-64

12 The Bellagio Study Group on Child Survival. Knowledge into action for child survival. Lancet 2003;362:323-7

13 [www.gatesfoundation.org/Global Health/Announcements/ Announce-050627.htm] accessed 29 June 2005

14 [http://gatesfoundation.org/default.htm] accessed 19 August 2005
15 Birn A-E. Gates's grandest challenge: transcending technology as public health ideology. Lancet 2005;366:514-19

16 [www.who.int/vaccines/GIVS/english/Global_imm._data_EN.pdf] accessed 19 August 2005

17 Greenwood BM, Bojang K, CJM Whitty CJ, Targett GA. Malaria. Lancet 2005;365:1487-98

18 [http://rbm.who.int/wmr2005/pdf/adv_e.pdf] accessed 19 August 2005

19 [http://www.unicef.org/specialsession/about/sgreport-pdf/02 ChildMalnutrition_D7341Insert_English.pdf] accessed 10 August 2005

20 Rudan I, Lawn J, Sousens S, et al. Gaps in policy-relevant information on burden of disease in children: a systematic review. Lancet 2005;365: $2031-40$

21 [www.gatesfoundation.org/globalhealth/breakthroughscience/ grandchallenges.htm] accessed 13 October 2005 